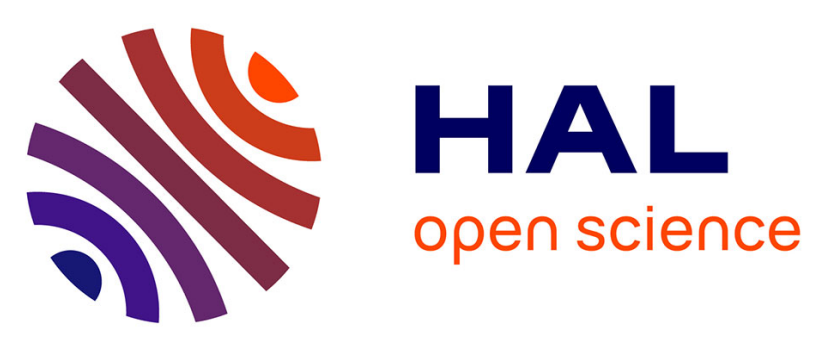

\title{
Comparison of MRI and motor evoked potential with triple stimulation technique for the detection of brachial plexus abnormalities in multifocal motor neuropathy
}

Giovanni Corazza, Thomas Le Corroller, Aude-marie Grapperon, Emmanuelle Salort-Campana, Annie Verschueren, Shahram Attarian, Emilien Delmont

\section{To cite this version:}

Giovanni Corazza, Thomas Le Corroller, Aude-marie Grapperon, Emmanuelle Salort-Campana, Annie Verschueren, et al.. Comparison of MRI and motor evoked potential with triple stimulation technique for the detection of brachial plexus abnormalities in multifocal motor neuropathy. Muscle \& Nerve, 2020, 61 (3), pp.325-329. 10.1002/mus.26773 . hal-02528251

\section{HAL Id: hal-02528251 \\ https://hal.science/hal-02528251}

Submitted on 7 Apr 2020

HAL is a multi-disciplinary open access archive for the deposit and dissemination of scientific research documents, whether they are published or not. The documents may come from teaching and research institutions in France or abroad, or from public or private research centers.
L'archive ouverte pluridisciplinaire HAL, est destinée au dépôt et à la diffusion de documents scientifiques de niveau recherche, publiés ou non, émanant des établissements d'enseignement et de recherche français ou étrangers, des laboratoires publics ou privés. 


\title{
Comparison of MRI and motor evoked potential with triple stimulation technique for the detection of brachial plexus abnormalities in multifocal motor neuropathy
}

\author{
Giovanni Corazza MD ${ }^{1}$ | Thomas Le Corroller MD PHD ${ }^{2}$ | \\ Aude-Marie Grapperon MD $^{1}$ ～Emmanuelle Salort-Campana MD $^{1}$ । \\ Annie Verschueren MD ${ }^{1}$ ～Shahram Attarian MD, PHD ${ }^{1}$ | Emilien Delmont MD, PHD ${ }^{1,3}$
}

\author{
${ }^{1}$ Referral Centre for Neuromuscular Diseases \\ and ALS, Hôpital La Timone, Marseille, France \\ ${ }^{2}$ Department of Radiology, Institute for \\ Locomotion, Hôpital Sainte-Marguerite, \\ Marseille, France \\ ${ }^{3}$ Aix-Marseille University, Timone \\ Neuroscience Institute, UMR CNRS 7289, \\ Marseille, France \\ Correspondence \\ Delmont Emilien, Referral center for ALS and \\ neuromuscular diseases, hospital La Timone \\ 264 rue Saint Pierre, 13005 Marseille, France. \\ Email: emilien.delmont@ap-hm.fr
}

\begin{abstract}
Background: Conduction blocks (CB) are the diagnostic hallmark of multifocal motor neuropathy (MMN). Conventional nerve conduction studies cannot detect $\mathrm{CB}$ above Erb's point. Our purpose was to compare the performance of the motor evoked potential with triple stimulation technique (MEP-TST) and MRI in the detection of abnormalities of the brachial plexus.

Methods: Examinations were performed on 26 patients with MMN (11 definite, 6 probable, 9 possible), of whom 7 had no $C B$.

Results: MEP-TST detected proximal CB in 19/26 patients. Plexus MRI showed T2 hyperintensity in 18/26 patients, with nerve enlargement in 14/18. A combination of both techniques increased the detection rate of brachial plexus abnormalities to $96 \%$ of patients (25/26).

Conclusions: MEP-TST and MRI have high sensitivities for detecting brachial plexus abnormalities. A combination of the two techniques increases the detection rate of supportive criteria for the diagnosis of $\mathrm{MMN}$.
\end{abstract}

\section{KEYWORDS}

brachial plexus, brachial plexus MRI, motor evoked potential, multifocal motor neuropathy with conduction blocks, proximal conduction block, triple stimulation technique

\section{1 | INTRODUCTION}

Multifocal motor neuropathy (MMN) is a chronic immune-mediated neuropathy. ${ }^{1}$ Conduction blocks (CB) are the main diagnostic hallmark. However, conduction failure is not always detected by

Abbreviations: ALS, amyotrophic lateral sclerosis; $\mathrm{CB}$, conduction blocks; CIDP, chronic inflammatory demyelinating polyradiculoneuropathy; CMAP, compound muscle action potential; EFNS/PNS, European Federation of Neurological Societies/Peripheral Nerve Society; IgM, immunoglobulin M; IVIg, intravenous immunoglobulins; MEP-TST, motor evoked potential with triple stimulation technique; MMN, multifocal motor neuropathy; MMN-RODS, Rasch-built Overall Disability Scale for Multifocal motor neuropathy; MRC Medical Research Council; ONLS, Overall Neuropathy Limitations Scale; PCB, proximal conduction block; STIR, short tau inversion recovery. conventional nerve conduction studies, ${ }^{2,3}$ especially in the presence of severe axonal loss, proximal $\mathrm{CB}(\mathrm{PCB})$ or distal $\mathrm{CB}$. The motor evoked potential with triple stimulation technique (MEP-TST) can detect PCBs located between the cervical roots and Erb's point. In one study, ${ }^{4}$ the MEP-TST improved the sensitivity of diagnostic criteria for definite or probable MMN from $60 \%$ to $90 \%$, providing considerable additional diagnostic power in comparison to classical nerve conduction studies.

Several additional criteria have been established to improve the diagnostic sensitivity of $\mathrm{MMN}$ including response to intravenous immunoglobulin (IVIg) therapy, positive immunoglobulin M (IgM) antiGM1 antibodies, and abnormal MRI of the nerves. ${ }^{1}$ Brachial plexus 
MRI scans may show nerve enlargement or an increased signal intensity on T2-weighted imaging. Few studies have focused on the importance of brachial plexus MRI in the diagnosis of $\mathrm{MMN}^{5-7}$ and brachial plexus MRI scans have not been compared with MEP-TST results in the context of MMN diagnosis.

The purpose of this study was to compare the effectiveness of the MEP-TST test and brachial plexus MRI in detecting plexus abnormalities in patients with $M M N$ with $C B$.

\section{2 | METHODS}

Patients were monitored at the Referral Centre for Neuromuscular Diseases of La Timone Hospital, Marseille, France. Patients with a diagnosis of MMN according to the 2010 European Federation of Neurological Societies/Peripheral Nerve Society (EFNS/PNS) criteria, ${ }^{1}$ who underwent brachial plexus MRI and MEP-TST tests between November 2017 and November 2018 were included in this retrospective study. Patients with central nervous system diseases or cervical root disorders that could have influenced MRI or MEP-TST test results were excluded. This study was approved by the ethics committee of the Assistance Publique des Hôpitaux de Marseille (2019_151). All patients gave informed consent. The MRI scans and MEP-TST tests were part of their routine evaluation. Sex, age, number of affected nerves, number of affected limb regions (range 0-8), Medical Research Council (MRC) sum-score (range 0-140), ${ }^{3}$ cerebrospinal fluid protein level, presence and titer of IgM anti-GM1 antibodies were recorded. Disability was assessed using the Overall Neuropathy Limitations Scale (ONLS) ${ }^{8}$ and the Rasch-built Overall Disability Scale for Multifocal Motor Neuropathy (MMN-RODS). ${ }^{9}$ IVlg treatment was considered effective if muscle weakness improved by at least 1 point on the MRC sum-score after 3 courses. ${ }^{3,10}$

Nerve conduction studies were performed on the median, ulnar, tibial, and fibular nerves. ${ }^{3}$ Radial and musculocutaneous nerves were not analyzed because their study is not systematic in our department. The fibular nerve was stimulated at the ankle, fibula head, and popliteal fossa, recording over the extensor digitorum brevis. The tibial nerve was stimulated at the ankle and popliteal fossa, recording over the abductor hallucis. The median nerve was stimulated at the wrist and elbow, recording over the abductor pollicis brevis. The ulnar nerve was stimulated at the wrist, below elbow, and above elbow recording over the abductor digiti minimi. Stimulation of the median and ulnar nerves was performed at the axilla and at Erb's point if no CB were detected in the forearm. To ensure that maximum stimulation was applied to the motor nerve at Erb's point, monopolar stimulation was used when necessary. Definite CB were defined as reductions of at least $50 \%$ of the compound muscle action potential (CMAP) area of the negative peak on proximal stimulation versus distal stimulation. ${ }^{1}$ Due to the difficulty in achieving supramaximal popliteal fossa stimulation, CB were not assessed in the tibial nerves. Antidromic sensory nerve action potentials were recorded from the median, ulnar, and sural nerves.

The MEP-TST tests were performed and recorded as previously described. ${ }^{11}$ The median and ulnar nerves were explored bilaterally with respective recordings on the abductor pollicis brevis and abductor digiti minimi muscles. Transcranial magnetic stimulation was followed by two supra-maximal electrical stimuli: one applied to the ulnar and median nerves at the wrist, and the other to the brachial plexus at Erb's point, at appropriate intervals. PCB was defined by an amplitude ratio below $90 \%{ }^{4}$ When PCB were suspected, repositioning, and three trials of the triple stimulation were performed to exclude technical artifacts.

Brachial plexus MRI examinations were performed on a PHILIPS Ingenia 1.5 T Omega ${ }^{\circledR}$ using the following sequences: 3DT1-weighted, 3DT2-weighted short tau inversion recovery (STIR), 3DT1-weighted with gadolinium enhancement. The plexus was examined bilaterally from the nerve roots to the axilla. The MRI images were interpreted by a single radiologist with 12 years of experience in musculoskeletal imaging who was not blinded to patient information. An abnormal MRI was defined either by nerve enlargement or T2 hyperintensity of the brachial plexus. ${ }^{5}$

Statistical analyses were performed using SPSS ${ }^{\circledR}$ (International Business Machines Corporation, Armonk, USA). Continuous data were expressed in median with interquartile ranges. Quantitative variables were compared using a Student t-test or a Mann-Whitney test, while qualitative data were evaluated using a Chi-squared test or a Fisher's exact test, according to the population distribution. A P-value below 0.05 was considered to be significant.

\section{3 | RESULTS}

A total of $47 \mathrm{MMN}$ patients came in the department between November 2017 and November 2018. Twenty-six of these patients underwent MEP-TST tests and plexus MRI during this period and were included in this study. Their characteristics are shown in Table 1. Eleven were classified as definite, six as probable, and nine as possible MMN. Among patients with possible $M M N$, seven patients had no history of $C B s$ during nerve conduction studies. ${ }^{3}$ All but two patients were on IVIg treatment at the time of the MRIs and MEP-TST testing. The median time from diagnosis to examination was 1 year (range, 0.5-4 years).

The MEP-TST tests were performed bilaterally on the median and ulnar nerves. The electrophysiological study could not be fully interpreted in 11 nerves due to distal CMAP amplitude below $1 \mathrm{mV}$. Thirtysix PCB were found in 19 of 26 patients (73\%), with a median of $1 \mathrm{PCB} /$ patient (Figure 1). Results by diagnostic category are given in Table 2. Eleven PCB were found in the right median nerves, 8 PCB in the left median nerves, 8 PCB in the right ulnar nerves, and 9 PCB in the left ulnar nerves. $\mathrm{PCB}$ were bilateral in 9/19 patients (47\%) and unilateral in 10/19 (53\%). All patients with normal MEP-TST results had muscle weakness in the region of at least one median or ulnar nerve, except for one patient who had only proximal weakness of the right arm.

Brachial plexus MRIs were abnormal in $18 / 26$ patients (69\%): STIR hyperintensity in 18 patients and nerve enlargement on T1-weighted imaging in 14 patients (Figures 1 and 2). Results by diagnostic category are given in Table 2. Gadolinium enhancement was never detected. Abnormalities were symmetrical and bilateral in $8 / 18$ patients (44\%), asymmetrical and bilateral in 5/18 (28\%), and unilateral in 5/18 (28\%). Patients with abnormal MRIs were younger, had more affected nerves and had higher titers of anti-GM1 antibodies (Table 1). 
TAB LE 1 Characteristics of the 26 patients with multifocal motor neuropathy and comparison between patients with normal and abnormal examination

\begin{tabular}{|c|c|c|c|c|c|c|c|}
\hline & $\begin{array}{l}\text { All patients } \\
n=26\end{array}$ & \multicolumn{3}{|c|}{ Brachial plexus MRI } & \multicolumn{3}{|l|}{ MEP-TST } \\
\hline Age (years) & $54(46-70)$ & 68 & 49 & $=.02$ & 50 & 61 & NS \\
\hline Male gender & 22 (81\%) & 7 & 15 & NS & 7 & 15 & NS \\
\hline Disease duration (years) & $3.5(2-10)$ & 2.75 & 5.5 & NS & 6 & 3 & NS \\
\hline Improvement after IVIg & $23 / 26(88 \%)$ & 6 & 17 & NS & 6 & 17 & NS \\
\hline Total score (/12) & $2(1-3)$ & 1.5 & 2 & NS & 3 & 2 & NS \\
\hline Upper limbs (/5) & $2(1-2)$ & 1 & 2 & NS & 2 & 1 & NS \\
\hline Lower limbs (/7) & $0(0-1)$ & 0 & 1 & NS & 0 & 1 & NS \\
\hline MMN-RODS (/100) & $72(60.5-92.7)$ & 83 & 72 & NS & 68 & 79 & NS \\
\hline Protein level in CSF (g/L) & $0.4(0.3-0.5)$ & 0.4 & 0.4 & NS & 0.4 & 0.37 & NS \\
\hline \multicolumn{8}{|l|}{ IgM anti-GM1 antibodies } \\
\hline Positive patients (\%) & $13(52 \%)$ & 2 & 10 & NS & 6 & 7 & NS \\
\hline Titer & $20(0-80)$ & $0(0-50)$ & $20(0-80)$ & $=.04$ & $80(60-720)$ & $0(0-60)$ & NS \\
\hline
\end{tabular}

Abbreviations: Quantitative data are expressed in median with interquartile ranges. Note: CSF, cerebrospinal fluid; NS, nonsignificant.

(A)

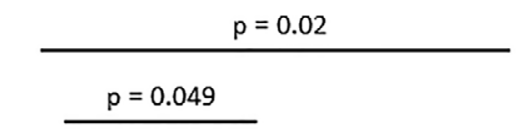

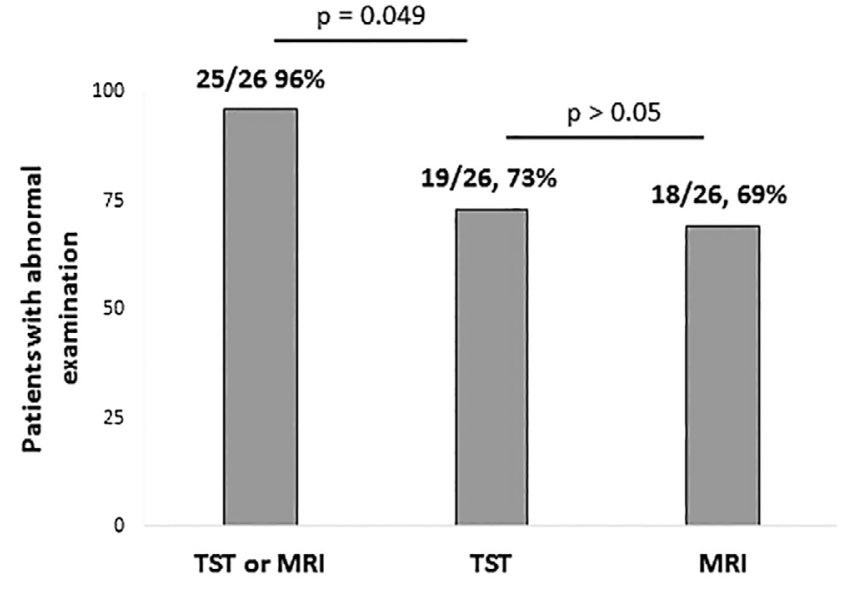

All MMN patients
(B) 100

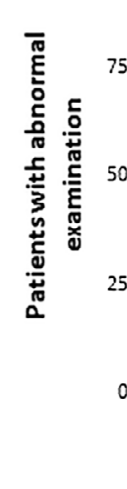

0 TST or MRI TST
MMN without conduction blocks on
routine nerve conduction studies

FIGURE 1 Number and percentage of patients with abnormal examinations. A, All 26 MMNs. B, Seven patients with MMN without conduction block on routine nerve conduction studies

TABLE 2 Results of brachial plexus MRI and MEP-TST according to diagnostic category

\begin{tabular}{|lllll}
\hline Diagnostic category & Definite & Probable & Possible & P-Value \\
\hline Abnormal brachial plexus MRI & $9 / 11(82 \%)$ & $3 / 6(50 \%)$ & $6 / 9(66 \%)$ & NS \\
\hline Abnormal MEP-TST & $8 / 11(73 \%)$ & $5 / 6(83 \%)$ & $6 / 9(66 \%)$ & NS \\
\hline
\end{tabular}

Note: NS, nonsignificant. 

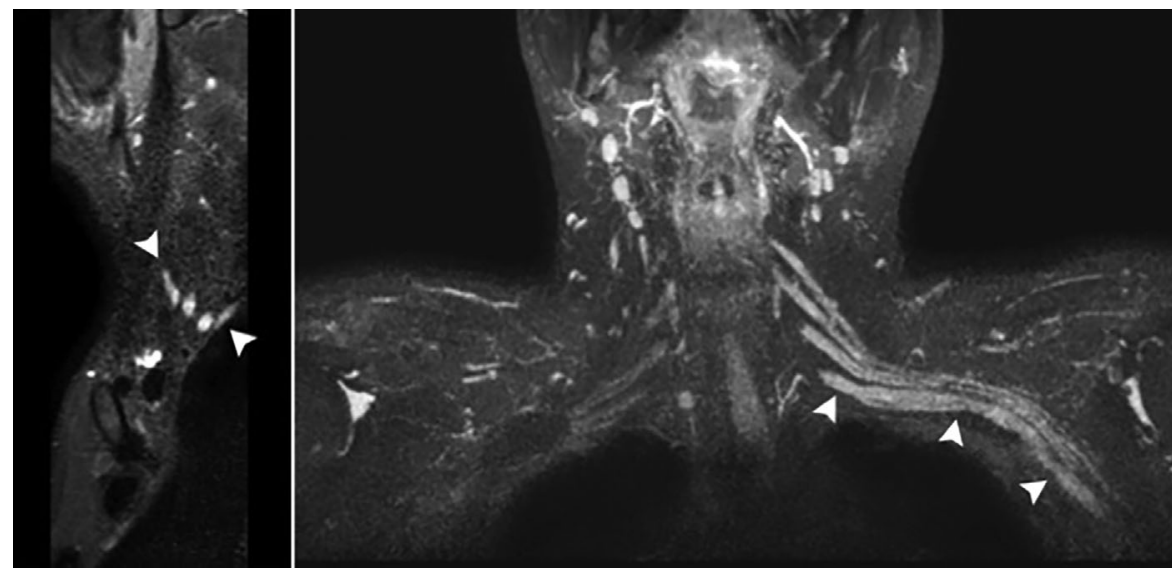

FIGURE 2 Brachial plexus MRI, sagittal reformatted STIR imaging, and coronal MIP STIR imaging displaying hyperintense signal (arrowheads) involving the brachial plexus on the left side. MIP, maximum intensity projection

The abnormal examination rate was similar for MEP-TST tests and plexus MRI (Figure 1), but eight patients had abnormal MEP-TST results and normal MRI, and six patients had normal MEP-TST results and abnormal MRI. Twenty-five patients had either an abnormal MEP-TST or an abnormal plexus MRI, which occurred more frequently than instances of an abnormal MEP-TST result alone or abnormal plexus MRI alone (Figure 1).

A subgroup of seven patients with possible MMN never had CB on several conventional nerve conduction studies. There was no significant difference in the detection rate of plexus abnormalities between MRI and MEP-TST tests. The detection rate for abnormalities was not significantly increased by the combination of the two techniques (Figure 1).

\section{DISCUSSION}

The demographic characteristics of our MMN patients were similar to those found in cohorts of previous studies ${ }^{12}$ : primarily middle-aged men with multifocal involvement of motor nerves, responsive to IVIg treatment and with positive anti-GM1 antibodies. Some MMN patients do not have history of $\mathrm{CB}$ following several nerve conduction studies. ${ }^{3}$ Such patients without CB were previously thought to have an axonal form of $M M N N^{2}$ However, the abnormalities of the brachial plexus observed following MEP-TST testing and MRIs were more indicative of MMN with PCB. A former study reported that patients with and without $\mathrm{CB}$ with classical nerve conduction studies shared the same clinical features and responses to $\mathrm{IVIg}^{3}$

The MEP-TST test can detect PCB in chronic inflammatory demyelinating polyradiculoneuropathy (CIDP), in Guillain-Barré syndrome, and in $\mathrm{MMN}^{4,11,13,14}$ This examination provides functional information and partly explains the origin of the muscle weakness experienced by the patients. The MEP-TST test helps to diagnose MMN, especially if $\mathrm{CB}$ are not detected in conventional nerve conduction studies. In this study, PCB were usually found in at least one of four nerves tested, and were unilateral approximately half of the time. This finding underscores the need for bilateral examination of the ulnar and median nerves. A limitation of the MEP-TST test is that it does not distinguish PCBs above Erb's point from the involvement of upper motor neurons. ${ }^{15}$ Other electrophysiological techniques such as silent period duration or cortico-motor excitatory thresholds may be performed by transcranial magnetic stimulation and can detect involvement of the central nervous system. ${ }^{16}$

Brachial plexus MRIs were abnormal in $35-63 \%$ of $M M N$ patients in previous studies, ${ }^{5-7,17}$ and in $69 \%$ of patients in our study. This difference may be explained by an improvement in MRI technique, by the fact that the MRI examinations were read by an experienced and unblinded radiologist, and because nearly half of our patients fulfilled the diagnostic criteria for definite MMN. When abnormal, brachial plexus MRI showed T2-hyperintensity in $86-100 \%$ of MMN patients, as well as nerve enlargement in $64-86 \%$ of such patients. In CIDP, plexus MRI detected nerve enlargement in $40-55 \%$ of patients, as well as T2-hyperintensity in $44-70 \%$ of such patients. ${ }^{6,7,18}$ Gadolinium enhancement has been reported in up to $36 \%$ of CIDP patients. ${ }^{18}$ However, it was not observed in our MMN patient cohort or in any from previous studies. ${ }^{6} \mathrm{MRI}$ abnormalities are usually more symmetrical in CIDP and asymmetrical in $M M N{ }^{7}$ A benefit of plexus MRI is that it can be informative, even in cases of severe muscle atrophy, while the MEP-TST test is not possible when distal CMAPs amplitudes are too low. The morphological abnormalities observed on brachial plexus MRIs are not specific for MMN. Two studies showed an increase in T2 signal intensity and brachial nerve root volume in some amyotrophic lateral sclerosis (ALS) patients. However, these abnormalities appeared to be less common than in patients with MMN and CIDP. ${ }^{17,19}$

Patients with normal and abnormal MEP-TST results had similar clinical features. On the other hand, more nerves were involved in patients with abnormal brachial plexus MRI. Because the MRC sum-score, number of affected regions, and disability scales were comparable in both populations, we do not believe that patients with an abnormal plexus MRI have a more serious disease. Patients with abnormal MRIs were also younger. However, these data should be considered with caution, as there was no difference in disease duration between the two groups. Furthermore, previous studies have not found a correlation between plexus MRI and disease progression. ${ }^{7}$

The MEP-TST tests and brachial plexus MRI showed abnormal features in $73 \%$ and $69 \%$ of MMN patients, respectively. The frequency of abnormal examination was similar in patients with definite, probable, and 
possible MMN, based on EFNS/PNS diagnostic criteria. ${ }^{1}$ Most patients were on IVIg therapy at the time of the investigations. Therefore, it can be assumed that IVIg did not interfere with the results of MEP-TST tests and plexus MRIs. However, this assertion should be confirmed in a longitudinal study, as well as in untreated patients. It can be hypothesized that the lack of gadolinium enhancement may be due to IVIg treatment, although a previous study conducted only in untreated patients did not show any abnormal gadolinium enhancement of the plexus. ${ }^{6}$ The contrast study can be helpful for the detection of alternative pathologies, such as malignant infiltration. In this study, the rate of abnormal examination may have been enhanced because the examiners were not blinded of the diagnosis of $M M N$.

The MEP-TST tests and plexus MRI have individually high sensitivities in this setting, and the combination of both techniques increases detection of abnormalities in MMN patients who meet EFNS/PNS diagnostic criteria. ${ }^{1}$ This study was not designed to analyze the specificity of the MEP-TST test and plexus MRI in the diagnosis of MMN. Further studies should collect longitudinal data and compare $\mathrm{MMN}$ patients with a control group.

\section{CONFLICT OF INTEREST}

None of the authors has any conflict of interest to disclose.

\section{ETHICAL PUBLICATION STATEMENT}

We confirm that we have read the Journal's position on issues involved in ethical publication and affirm that this report is consistent with those guidelines.

\section{REFERENCES}

1. European Federation of Neurological Societies/Peripheral Nerve Society guideline on management of multifocal motor neuropathy. Report of a joint task force of the European Federation of Neurological Societies and the Peripheral Nerve Society--first revision J Peripher Nerv Syst. 2010;15:295-301.

2. Katz JS, Barohn RJ, Kojan S, et al. Axonal multifocal motor neuropathy without conduction block or other features of demyelination. Neurology. 2002;58:615-620.

3. Delmont E, Azulay JP, Giorgi R, et al. Multifocal motor neuropathy with and without conduction block: a single entity? Neurology. 2006; 67:592-596.

4. Deroide N, Uzenot D, Verschueren A, Azulay JP, Pouget J, Attarian S Triple-stimulation technique in multifocal neuropathy with conduction block. Muscle Nerve. 2007;35:632-636.

5. Van Es HW, Van den Berg LH, Franssen H, et al. Magnetic resonance imaging of the brachial plexus in patients with multifocal motor neuropathy. Neurology. 1997;48:1218-1224.

6. Goedee HS, Jongbloed BA, van Asseldonk JH, et al. A comparative study of brachial plexus sonography and magnetic resonance imaging in chronic inflammatory demyelinating neuropathy and multifocal motor neuropathy. Eur J Neurol. 2017;1307-1313.

7. Jongbloed BA, Bos JW, Rutgers D, van der Pol WL, van den Berg LH. Brachial plexus magnetic resonance imaging differentiates between inflammatory neuropathies and does not predict disease course. Brain Behav. 2017;7:3-7.

8. Graham RC, Hughes RAC. A modified peripheral neuropathy scale: the Overall Neuropathy Limitations Scale. J Neurol Neurosurg Psychiatry. 2006;77:973-976.

9. Vanhoutte EK, Faber CG, van Nes SI, et al. Rasch-built Overall Disability Scale for Multifocal motor neuropathy (MMN-RODS). J Peripher Nerv Syst. 2014;20:296-305.

10. Pruppers MHJ, Draak THP, Vanhoutte EK, et al. Outcome measures in MMN revisited: further improvement needed. J Peripher Nerv Syst. 2015;20:306-318.

11. Attarian S, Azulay JP, Verschueren A, Pouget J. Magnetic stimulation using a triple-stimulation technique in patients with multifocal neuropathy without conduction block. Muscle Nerve. 2005;32:710-714.

12. Cats EA, Van Der Pol WL, Piepers S, et al. Correlates of outcome and response to IVIg in 88 patients with multifocal motor neuropathy. Neurology. 2010;75:818-825.

13. Attarian S, Franques J, Elisabeth J, et al. Triple-stimulation technique improves the diagnosis of chronic inflammatory demyelinating polyradiculoneuropathy. Muscle Nerve. 2015;51:541-548.

14. Sevy A, Grapperon AM, Salort Campana E, Delmont E, Attarian S. Detection of proximal conduction blocks using a triple stimulation technique improves the early diagnosis of Guillain-Barré syndrome. Clin Neurophysiol. 2018;129:127-132.

15. Attarian S, Verschueren A, Pouget J. Magnetic stimulation including the triple-stimulation technique in amyotrophic lateral sclerosis. Muscle Nerve. 2007;36:55-61.

16. Attarian S, Azulay JP, Lardillier D, Verschueren A, Pouget J. Transcranial magnetic stimulation in lower motor neuron diseases. Clin Neurophysiol. 2005;116:35-42.

17. Staff NP, Amrami KK, Howe BM. Magnetic resonance imaging abnormalities of peripheral nerve and muscle are common in amyotrophic lateral sclerosis and share features with multifocal motor neuropathy. Muscle Nerve. 2015;52:137-139.

18. Fargeot $\mathrm{G}$, Viala K, Theaudin M, et al. Diagnostic usefulness of plexus MRI in chronic inflammatory demyelinating polyradiculopathy without electrodiagnostic criteria of demyelination. Eur J Neurol. 2019;26:631-638.

19. Gerevini S, Agosta F, Riva N, et al. MR Imaging of brachial plexus and limb-girdle muscles in patients with amyotrophic lateral sclerosis. Radiology. 2016;279:553-561. 